\title{
Study of Skeletal Remains: Solving a Homicide Case with Forensic Anthropology and Review of the Literature
}

\author{
Laura Donato $^{1}$, Alessandro di Luca ${ }^{2 *}$, Carla Vecchiotti ${ }^{1}$ and Luigi Cipolloni ${ }^{1}$ \\ ${ }^{1}$ Department of Anatomical, Histological, Forensic and Orthopaedic Sciences, University of Rome Sapienza, Viale Regina Elena 336,00161 Rome, Italy \\ ${ }^{2}$ Department of Public Health, University "SacroCuore" of Rome, Largo F. Vito 1, 00168 Rome, Italy
}

"Corresponding author: Alessandro di Luca, Department of Public Health, University "SacroCuore" of Rome, Largo F. Vito 1, 00168 Rome, Italy, Tel: +39/3338649213; E-mail: Aless.diluca@libero.it

Received date: June 8, 2016, Accepted date: June 20, 2016, Published date: June 30, 2016

Copyright: (C) 2016 Laura D, et al. This is an open-access article distributed under the terms of the Creative Commons Attribution License, which permits unrestricted use, distribution, and reproduction in any medium, provided the original author and source are credited.

\begin{abstract}
The present study examines a case in which signs of incised wounds were found on skeletal remains of a murder victim. The authors have studied the skeletal remains and the dynamics of the murder in order to reconstruct the kind of weapon utilized. The bones examined belonged to a woman that had disappeared from almost 10 years and where recovered from the garden of the house belonging to her former partner. The main feature of these remains was the discovery of a damage of a thoracic vertebra (T1). Our examination has the objective to enhance the macroscopic analysis of the skeletal remains in order to deduce information about the classification of specific damages and the dynamics that have caused them and the identification of the weapon that had been used. Highly decomposed bodies, as in skeletal remains, having poor or absent biological tissue on it, challenge the operator to classify the exact nature of the damage, and in some cases, it does not allow achieving a significant level of certainty. In order to solve this critical situation, Forensic Anthropology may contribute greatly by supplying a great amount of information that would not be deciphered otherwise. The bone, main study object of the Forensic Anthropology, may also register, as the soft tissues, the features of the damaging pattern. The opportunity to extrapolate this kind of data, allows analysing the dynamics and the nature of the kind of weapon used. In certain cases the incised bone present also features that allow to identify the exact structure of the weapon: of course, not every wound damages the bone, but when this actually happens, the morphological appearance of the instrument utilized remains crystallized in time, excluding of course the cases in which the bones are destroyed. Many other studies, concerning the characterization of lesions due to sharp objects, have been done and a review of related literature has been included in this article. The major goal of the authors is to highlight the importance of the information that can be extrapolated: the usefulness of the classification of the weapons used to provoke the lesion, could reach a more accurate evaluation in order to significantly help in case of forensic assessment.
\end{abstract}

Keywords: Forensic anthropology; Homicide; Bones, Cold case; Stab wound

\section{Introduction}

When the object of forensic investigation is constituted by skeletal remains, obtaining information is more difficult because of the absence of soft tissues. The source of information is represented by bones only. Certainly there are some missing tiles that can't be regained: many lesions or trauma that involves soft tissues don't involve bones and this is why the pathologist is the only one that can suggest the cause of death. The most important information is obtained by the study of lesions, and what kind of object caused it. A forensic anthropologist can state that there are some potentially fatal lesions. Yet, without soft tissues, it is not possible to gain certainty about the presence of other lesions, not involving bones that could have caused the death.

Whereas there are some objective limits in a forensic anthropological analysis, the item of its study provides interesting features that can be useful in a forensic contest. Strong tissues, made of organic and inorganic components, which involve a particular resistance and hardness, constitute bones. When this kind of tissues is involved it is functional to extrapolate the maximum information from the only present element. It does not matter if the soft tissues are absent or if the crime scene had been contaminated, bones keep the signs of any trauma or lesion and, furthermore, they keep the shape of the item that causes it.

\section{The Case}

In March 2001, in Rome, a woman went missing, and although extensive police investigation where performed, it was not possible to trace her. Her partner was questioned but the case was closed without achieving satisfactory results. In the subsequent years the case was opened once more by impulse of the "Cold Case" Unit of the Public Prosecutor's Office of Rome, and the investigations where focused on the partner of the victim, who was known to have a sentimental relation with the woman at the time where she disappeared. The man was questioned by the police and confessed that the woman had died the same day of her disappearance, indicating the place where he had buried the corpse. This first dig uncovered only the bones of several small animals, so the man was questioned once again, and finally confessed that the woman had died in his own home after a fight, when he had hit the victim causing her to lose balance, fall, and hit with the head a fireplace made of crude stone. The man also confessed that he had buried the woman's body in his own garden. This new dig, performed in 2009, allowed the retrieval of the bones of the deceased woman that where partially buried under a concrete wall, built during the past few years, delimiting the man's propriety. The body was still 
contained in the remains of the clothes that she was wearing at the time of her death. The bones where cautiously collected and brought to the Department of Legal Medicine in Rome for further investigations and analysis.

\section{Results}

As confirmed by the partner of the victim, the remains were located in the garden outside his house. In order to allow the retrieval, it was necessary to remove a layer of cement covering the "grave" soil. The mechanical means employed provoked the partial fragmentation of the skeletal remains, particularly the skull, and maxillary and zygomatic bone (Figure 1). After removing the cement layer, the excavation was carried on through proper means, following the forensic anthropological guidelines, in order to recover skeletal remains. The "grave" soil was removed using brushes to avoid damage the human remains, to observe the disposition of bones and to recover all of the part of the skeleton present inside the grave. Most of the skeletal elements where retrieved, except for bones of hands and feet. The absence of these parts was due to previous construction and maintenance work processed near the burial site in the previous years.

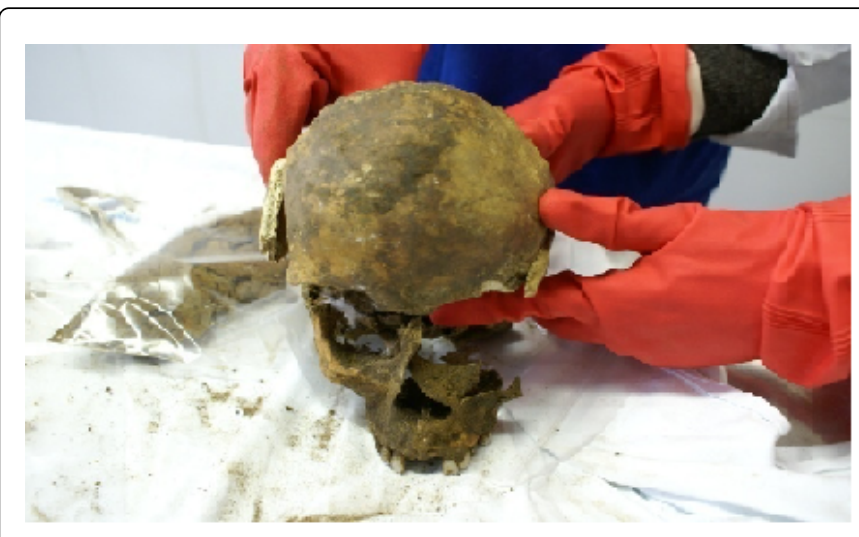

Figure 1: Anterior view of the skull with fragmented surface related to orbital and zygomatic bones.

An accurate study of every bone was performed in order to search eventual signs of lesion. A lesion at the level of the body of a thoracic vertebra was detected, with a small fragment still in place. When the fragment was removed, the surface of the vertebral body was clear and its colour differed from the rest of the bone element (Figure 2).

The lesion has been chronologically identified as peri-mortem since, at the moment of the medico-legal investigation, it was covered by a slice of bone that was cautiously removed during the study of the bone, showing a different colour compared to the rest of the vertebra. The chromatic difference was caused by the slice of the cut bone still sticking on the bony surface thanks to presence of the soft tissues and, after decomposition, to the pressure of the soil. The lesion showed a lighter colour: the colour of the bone surface depends on the environment in which the skeletal elements are preserved. Part of the components of the soil is absorbed by the surface of the bones, homogeneously modifying their colour. In this case, the lesion was classified as peri-mortem since the presence of a chromatic difference between the external surface and the lesion's surface colour. This difference was due to the gradually replacement of the soft tissues by the grave soil. Its pressure avoided the infiltration of soil components in the lesion, hence, the modification of the bone colour [1].

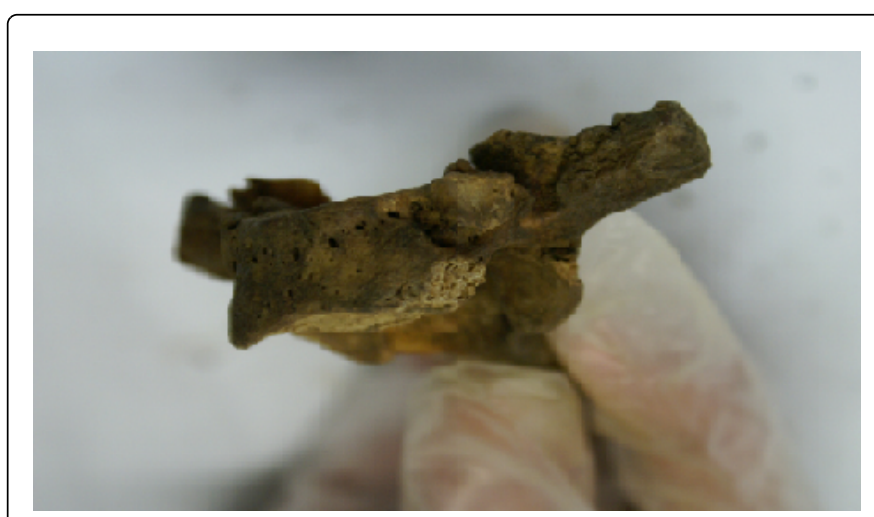

Figure 2: Vertebral body with incised surface on the lower right region of the body.

The skeletal elements where processed through forensic anthropological examination. Once identified the human morphology of these elements, through macroscopic inspection, further examination have been carried on in order to determine sex, age at death, stature and all pertinent features useful for the identification of the victim.

In order to facilitate the examination, the bones have been cleaned, put in anatomical order, and photographed: this allowed to state that the skeletal elements and their number belonged to a single individual (Figure 3). No fragmentation was detected, with the exception of the skull: it was hardly damaged because of the action of mechanical means employed to remove the soil and cement layers covering the skeletal remains (Figure 1). The fragmented skull allowed detecting Caucasoid traits. Useful feature for sex determination were appreciable: the shape of the maxillary bones and the supraorbital ridge were peculiar to a female. This information was added to the study of the pelvis: the presence of the ventral arch, the shape of the greater sciatic notch and the pelvic girdle were all significant elements confirming the diagnosis of female character.

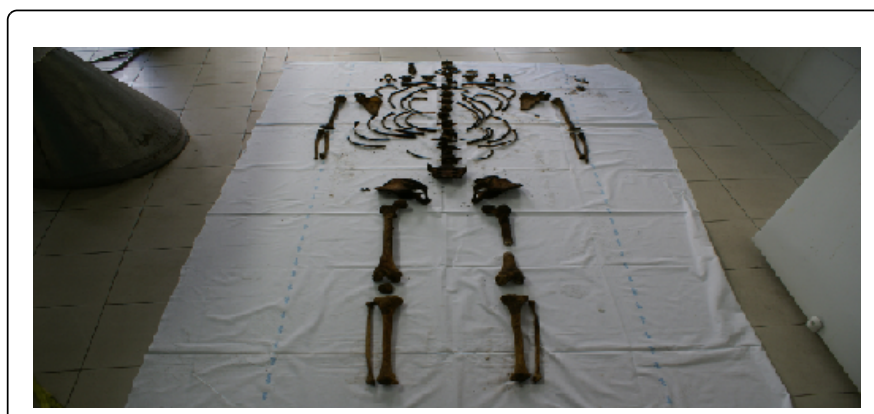

Figure 3: Post-cranial skeletal elements: bones of hands and feet have not been found during the recovery.

The absence of fragmentation of the post-cranial elements allowed observation of the level of maturation of bones in order to perform the evaluation of the age at death: long bone have completed their process of formation and reached their final shape, indicating a minimum age limit of 20-22 years. The topography of the auricular surface showed 
the characteristic irregularities related to a transition between phase 7 and phase 8 , referred to an age between 50 and 60 years [2]. The level of modification of pubic symphysis was compatible with phase 10 , which is more than 50 years. These evaluations agree to a diagnosis of age at death between 50 and 60 years [3].

The stature was estimated measuring femur and applying the equation proposed by Trotter [4]: 2.47 x 38 (femur length) $+54.10=147.96 \mathrm{~cm} \pm 3.72$

No markers related to occupational stress was found, only an initial phase of osteoarthritis observed on the bodies of two thoracic vertebrae.

The examination of the bones allowed detection of evidence of previous healed lesions.

The chromatic homogeneity of the fracture edges of the mandible and its surface gave relevant information about the timing this lesion, presuming it to correspond to the moment of death (Figure 4). Substantial difference was observed between the vertebral lesion and the mandibular fracture. The vertebral lesion showed chromatic difference because of the presence of the bone layer covering the cut surface still sticking thanks to the presence of the soft tissues and of the soil's pressure. The chromatic homogeneity between mandibular surface and its fracture edges, confirmed that the fracture was compound at the moment of the burial. The internal part of the mandibular bone was exposed, allowing the soil to be absorbed.

The forensic anthropological examination was fundamental to detect the suspect of identity. The results about age at death, sex and stature were compatible with the biological profile of the missing woman. In order to confirm the identity the victim a DNA test was performed and confirmed the identification of a 54 years old woman thanks to the genetic compatibility between her bones and her relative's genetic samples.

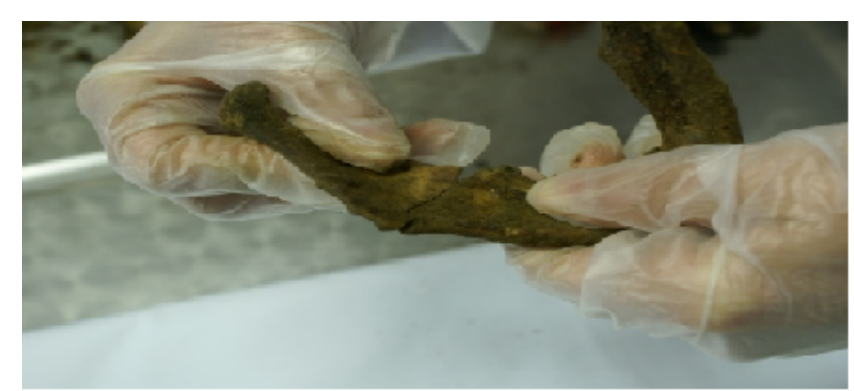

Figure 4: Mandible with fragmentation of the left ramus.

\section{Discussion}

In the exposed case the medico legal and forensic anthropological analysis allowed the highlight of a vertebral and mandibular lesion, classifying them as vital.

Particularly the lesion of the vertebral body permitted to state that it might have been provoked by the action of a blade. This permitted to reconstruct the dynamic of the homicide, attributing the death to an incised wound. Said wound damaged the vascular and nervous structure of the neck through the impact of the blade on the vertebral body.
This discovery allowed to confirm a first-degree murder accusation, and not, as previously stated by the murderer of an accidental death. The man was brought to trial and condemned due to the evidence found during the investigations.

In order to identify the kind of tool used to provoke the lesion, it was been necessary to review all the methods that have been employed in this kind of case files and to choose the most suitable to the actual case. The characteristics of a sharp tool are in fixed on the hard tissue, such as bone, and allow detecting its original shape, striations and the angle of impact [5]. The major goal of this study is to obtain the most possible accurate characterization of the murder weapon. An interesting aspect is how the shape of the lesion can give information about the dynamic of the injury: the direction of the blow, either if it was dealt perpendicularly or not, leaves a distinguishable mark. Many papers have been issued about $3 \mathrm{D}$ digital microscope to utilized to gain a three dimensional rendering of the analysed sample, that allow to measure and observe the characteristic striation left by particular kind of tools $[5,6]$. Using these techniques it is possible to evaluate the shape and direction of the wound and also the height from which the knife was handled [5].

Complementarily to this research, a qualitative analysis was realized using a technique based on the employment of the SEM/EDS method. The characteristic of SEM is to allow a detailed vision of the surface of the sample, while the EDS technique consent to detect different elements of the lesion, that have been left by the tool used to provoke the injury: the postulate is that bone, metal and organic material respond with a different brightness related to a gray scale. Metallic elements detected on the surface could be linked to the weapon used and could be deeply useful to supply peculiar data to accurately detect the object. Although EDS could deliver results with a strong probative value, it is subject to a detection limit that hinders it to deeply discriminate different type of steel $[7,8]$.

In some cases, the reliability of the Discriminant Function Analysis was evaluated for the differentiation of metal edges. The starting point is to realize the impression of the cut marks using a silicone based dental impression in order to observe it with a microscope and rebuild its $3 \mathrm{D}$ volume by scan [9].

It is also possible to distinguish the kind of sharp tool used to provoke the injury: for example, the lesion may be compared to the wound due to the action of a saw. In this particular case, the lesion would show a particularly different aspect, characterized by multiple cutting edges: in the analyzed case file, the edges of the wound where well defined and allow recognizing a single stab wound [10-14].

In order to evaluate the accuracy of identification of different kinds of lesions, incisions and other marks due to particular tools, analysis like blind or comparative tests have been realized employing SEM, Micro CT-scan and Epifluorescence Macroscopy [15-18]. In particular, Epifluorescence Macroscopy supplied accurate information characterizing different kinds of tools and providing high resolution data with non-destructive three dimensional visualization of the sample $[18,19]$. Concurrently, the acquisition of a three dimensional shape of a lesion employing the 3D Optical Digitalizer had performed better results about measurement efficiency, with an error rate of 0.05 $\mathrm{mm}$, and speed than SEM and CT systems. This technique, allows performing 3D image acquisition with particular accuracy in terms of soft tissue lesions [18].

Other variables have been considered useful to a correct identification of the tool: the resistance due to the presence of cloths 
had been object of studies. The presence of an outfit, as well as type of tool and anatomical location of the lesion, has a considerable effect in reduce the magnitude of penetrating forces [20].

SEM/EDS method had been used to evaluate the correspondence between the observed injury and the presumed weapon: three different cases had been analysed in Netherlands. During the examination of skeletal remains, the morphology of a lesion had been misinterpreted as a gunshot injury because of its features: circular wound with diameter of $15-19 \mathrm{~mm}$, attributable to large calibre ammunition. The employment of SEM/EDS allowed finding no gunshot residues on the bone surface: it highlighted the presence of other elements, corresponding to the chemical composition of a metal rod found near the skeletal remains, related to a bicycle lock. In case of injuries due to knife action, particles of the tool can remain on the involved surface due of the impact: these elements can be distinguished through the support of the SEM/EDS [7].

The employment of X-ray examination on skeletal remains could be a useful support for the diagnosis of murder. In spring 1994 skeletal remains have been recovered in a Danish forest. Most of the bones showed incisions: cervical column (the left transversal process of a cervical vertebra body), sternum (two incisions, one perforating the manubrium and the other located at the distal part of the sternum), iliac crest (perforating lesion), ribs (six ribs were incised, two of which were perforated), right tibia (proximal region showed two superficial wounds). Pelvic girdle and skull revealed male characteristics and the stature was estimated by measuring homerus and femur $(170 \mathrm{~cm})$. The skeletal elements were exposed to X-ray examination: no age-related degenerative changes were found but the lesions on ribs and iliac crest where highlighted. These skeletonized remains of a male of 25-30 years where identified with a disappeared 28-years Iranian man with height of $171 \mathrm{~cm}$. The autopsy declared that he was stabbed to death. In this case the X-ray examination allowed observing the incisions on the skeletal elements in order to identify the possible tool that provoked them [21].

It is possible to recognize the type of tool responsible for an incision also when this is located on ribs cartilage. 21 years old was stabbed in the chest, the abdomen and in the back: the lesions showed sharp and blunt corner and sharp margins with depth of 0.5 to $10 \mathrm{~cm}$. Several lesions were located on the costal cartilage and their surface showed evident striae due to the irregularity of the cutting edge. The shape of some lesions was linked to a curved abrasion located on the skin: this pattern highlighted the evidence of a particular shape between the blade and the grip. This information, in addiction to wounds shape related to a serrated blade, helped to identify and recover the item. All these lesions, particularly on cartilage, were observed with naked eye and by examination under polarized light microscope with low magnification. A dental impression medium was employed to reproduce the superficial structure of the lesion in order to perform direct comparison between knife standards and the surface of the wounded cartilage. To compare the reproduced surface, a standard knife cut impression was reproduced, by experimentally stabbing the knife in a brand of cellulose acetate butyrate. Differences between classes of objects are related to class and individual characteristics. Class characteristics refer to repetitive structural details on the surface. Individual characteristics depend on individual use. Defects on the cutting edge are generally well identified on bone: however, the cartilage could record finer details. In this case, all these exams highlighting the stab wound characteristics allowed to identify the weapon as a marine survival knife with a serrated blunt edge: the shape and defects of the blade were compatible with the lesions [22].

Bone remains are often considered the most difficult and challenging to be investigated: since the complexity of the case increases as more time passes since the moment of the death. It is important not to undervalue the available data: even if the only information is a photograph, some data could still be extrapolated. In this case, the features of the vertebral body (Figure 4) allow making a discrimination of the type of tool used and the dynamic of the blow. The first step was about to compare this kind of lesion to the wound provoked by different classes of tools. Three classes have been considered: saw hatchet and knife.

The first class describes a lesion provoked by the action of a saw is characterized by particularly diagnostic feature: multiple cutting edges, nearly parallel one to each other are due to the several passage of the numerous teeth of the saw. In this case there are no signs of multiple cutting edges [9]. Moreover, it is quite impossible that this kind of tool could damage just one vertebral body, considering that the incision in tangent to the body and not perpendicular: a saw would probably injure other nearby bone element [23].

The second class is constituted by the lesions occurring in case of a wound due to the action of a hatchet. A considerable number of damages can be produced: the impact site is characterized by possible bisection of the bone, the development of fractures, irregular edges of the lesion [13]. The sample under consideration displays regular edges, no fractures developing from the impact site or cracking of the vertebral body. As explained about saw lesion, it is nearly unfeasible for this kind of object to provoke this kind of incision without damaging other bones, because of the angle of impact.

The shape of the incision under examination has been considered to be more compatible with the third class of possible weapon: that is a lesion due to the action of a knife. A huge amount of different blades are accessible in the free market, as well as a great amount of other similar objects capable of killing, and their similarities make the difficult to distinguish one from the other just by the study of the incision (Figure 5). Anyway it is possible to discriminate two kinds of knife: serrated and non-serrated. Both these weapons deal linear and superficial incisions but there are peculiar features that can help to distinguish between them.

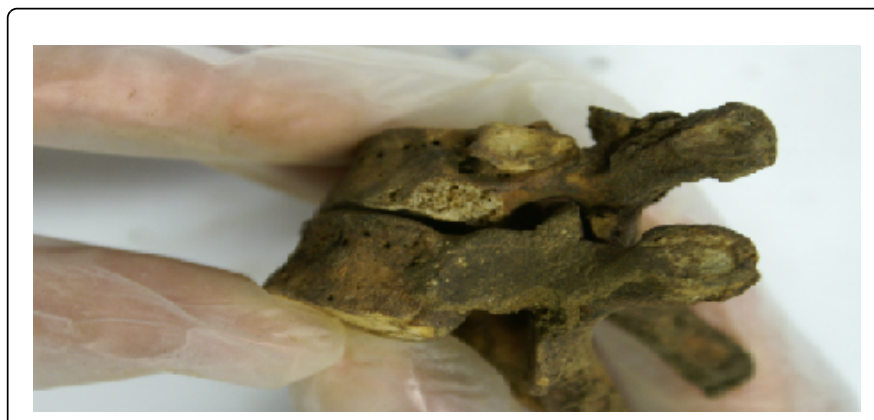

Figure 5: T1 and T2: the lesion is evident on C7, whereas the body of $\mathrm{T} 1$ shows no incised surface.

Serrated blades cause deeper and larger lesions to the bone surface and cause the production of fragments. 
Non serrated blade lesions are more superficial and no bone fragments are produced. The effect of this blade is to slice the surface of the bone: this was compatible with the effect showed by the lesion of the examined vertebral body, characterized by a superficial incision due to the slicing effect of a blade. A non-serrated blade has been considered suitable for the kind of analysed incision [24].

The weapon involved in the injury was provided of a sharp blade, this is deducible because of the shape of the lesion and its well defined edges.

There are two different hypotheses about the size of the blade:

The width of the incision corresponds to the blade's width: this means that it wasn't larger than $1 \mathrm{~cm}$ otherwise it would have marked and incised the nearby vertebral bodies.

The blade had a width greater than $1 \mathrm{~cm}$ : the incision had been provoked by the blow involving only the inferior edge of the blade.

In order to reconstruct the dynamic of the action it is necessary to detect:

The anatomical position of the bone,

The shape of the incision,

Other incisions on nearby bones,

The incision is located on the left side of a thoracic vertebral body (T-1).

The shape of the incision is particularly important to determine the direction of the wound. In this case it's possible to observe a rounded edge, near the transverse process, indicating the direction of the blow.

The incision is characterized by a sharp edge on the opposite side of the rounded bound and it goes along the surface of the vertebral body. The shape of the sharp edge is indicative of the point where the blade stopped and, therefore, revealing the direction of the wound.

There are no other incisions on the nearby vertebrae.

According with these results, it's possible to reconstruct the dynamic of the wound: the offender was located on the back (relatively lateral) of the victim and the wound was tangent to the left side of the vertebral body, moving from the posterior to the anterior side of the vertebra. The incision corresponds to a single wound.

\section{Conclusions}

The accurate analysis of skeletal remains showing cut marks or any other kind of lesion, are fundamental when soft tissues are absent or badly preserved $[25,26]$. A wide range of analysis can be employed and the evaluation of the results is object of a forensic anthropological study: the information that can be extrapolated could be critical in the process of identifying the class of tool, employed to perform the lesion. Microscopic and macroscopic exams are tested in order to execute a reproducible and accurate diagnosis: X-ray examination, SEM-EDS, polarized light microscope, naked eye are some of the means used to perform the analysis.

A general review of the skeletal elements is the first step: through the observation with naked eye an initial overview of the condition of the bones is fundamental. The detection of eventual lesions and their position could supply a starting point that can aim the selection of the appropriate exam in order to highlight and measure the lesion.
It isn't always possible to perform particular kind of analysis, as SEM-EDS for example, because of availability and cost of the means. Performing a naked eye examination it is also achievable to collect important information: the localization of the anatomical district showing the lesion could suggest an approximated dynamic of the blow and the macroscopic shape of the incision can be linked to a particular kind of impact [27,28].

The description of the wound has to consider nearby the skeletal elements: fractures expanding from the point of impact or close faint incisions are patterns that can increase the explicative value of the principal wound [29-32]. The level of involvement of the nearby anatomic elements, the importance and the configuration of the lesion differentiate the weapon. Once the class of the weapon has been found, a more elaborated and accurate description can be realized making use of a polarized light microscope: the edges of an incision, their shape and size represent the negative imprint of a blade. Microscopically observing the pattern of the wound can supply peculiar details of the tool, due to imperfections of the blade or particular shape: this information, in addiction to measurements of depth and width, collimate to describe the detected tool as accurately as possible. When skeletal elements conditions allow to perform these kind of analysis, a forensic anthropologist can obtain information that need to be submitted to the medico-legal evaluation in order to eventually diagnose the cause of death.

\section{References}

1. Coelho L, Cardoso HF (2013) Timing of blunt force injuries in long bones: the effects of the environment, PMI length and human surrogate model. Forensic Sci. Int. 233: 230-237.

2. Lovejoy CO, Meind RS, Pryzbeck TR, Mensforth RP (1985) Chronological metamorphosis of the auricular surface of the ilium: A new method for determination of adult skeletal age at death. American Journal of Physical Anthropology 68: 15-28.

3. Todd TW (1920) "Age changes in pubic bone: I. The white male pubis. American Journal of Physical Anthropology 3: 467-470.

4. Trotter M (1970) Estimation of stature from intact long bones. In: TD. Stewart. Personal identification in mass disasters pp: 71-83.

5. Shaw KP, Chung JH, Chung FC, Tseng BY, Pan CH, et al. (2011)A method for studying knife tool marks on bone. J Forensic Sci 56: 967-971.

6. Bello SM, Soligo C (2008) A new method for the quantitative analysis of cutmark micromorphology. Journal of Archaeological Science 35: 1542-1552.

7. Vermeij EJ, Zoon ED, Chang SB, Keereweer I, Pieterman R, et al. (2012) Analysis of microtraces in invasive traumas using SEM/EDS. ForensicSci Int 214: 96-104.

8. Gibelli D, Mazzarelli D, Porta D, Rizzi A, Cattaneo C (2012) Detection ofmetal residues on bone using SEM-EDS--part II: Sharp force injury. Forensic Sci. Int 223: 91-96.

9. Bonney H (2014) An investigation of the use of discriminant analysis for the classification of blade edge type from cut marks made by metal and bamboo blades. Am. J. Phys. Anthropol 154: 575-84.

10. Freas LE (2010) Assessment of wear-related features of the kerf wall from saw marks in bone. J. Forensic Sci 55: 1561-1569.

11. Kemal CJ, Patterson T, Molina DK (2013) Deaths due to sharp force injuries in Bexar County, Texas, with respect to manner of death. Am. J. Forensic Med. Pathol 34: 253-259.

12. Saltzherr TP, Fung KonJin PH, Beenen LF, Vandertop WP, Goslings JC (2009) Diagnostic imaging of cervical spine injuries following blunt trauma: a review of the literature and practical guideline. Injury 40 : 795-800. 
Citation: Donato L, di Luca, Vecchiotti C, Cipolloni L (2016) Study of Skeletal Remains: Solving a Homicide Case with Forensic Anthropology and Review of the Literature. J Forensic Anthropol 1: 105.

Page 6 of 6

13. Kaliszan M, Karnecki K, Tomczak E, Gos T, Jankowski Z (2013) Complex suicide by self-stabbing with subsequent drowningin the sea J. Forensic Sci 58: 1370-1373.

14. Delannoy Y, Colard T, Becart A, Tournel G, Gosset D, Hedouin V (2013) Typical external skull beveling wound unlinked with a gunshot. Forensic. Sci. Int 226: 4-8.

15. Bashir M, McWilliams RG, Desmond M, Kuduvalli M, Oo A, Field M (2013) Blunt aortic injury secondary to fragmented tenth thoracic vertebral body. Ann. Thorac. Surg 95: 2161-2164.

16. Ambade VN, Godbole HV (2006) Comparison of wound patterns in homicide by sharp and blunt force"Forensic Sci. Int 156: 166-170.

17. Blumenschine RJ, Marean CW, Capaldo SD (1996) Blind Tests of Interanalyst Correspondence and Accuracy in the Identification of Cut Marks, Percussion Marks, and Carnivore Tooth Marks on Bone Surfaces. Journal of Archaeological Science 23: 493-507.

18. Pechníková M, Porta D, Mazzarelli D, Rizzi A, Drozdová E, et al. (2012) Detection of metal residues on bone using SEM-EDS. Part I: Blunt force injury. Forensic Sci. Int 223: 87-90.

19. Capuani C, Rouquette J, Payré B, Moscovici J, Delisle MB, et al. (2013) Deciphering the elusive nature of sharp bone trauma using epifluorescence macroscopy: acomparison study multiplexing classical imaging approaches. Int. J. Legal Med 127: 169-176.

20. Sansoni G, Cattaneo C, Trebeschi M, Gibelli D, Porta D, et al. (2009) Feasibility of contactless 3D optical measurement for the analysis of bone and soft tissue lesions: new technologies and perspectives in forensic sciences. J. Forensic Sci 54: 540-545.

21. Poulsen LW, Vesterby A (1997) The diagnosis of a murder from skeletal remains: a case report. Int. J. Legal Med 110: 97-100.

22. Rao VJ, Hart R (1983) Tool mark determination in cartilage of stabbing victim. J. Forensic Sci 28: 794-799.
23. Capuani C, Guilbeau-Frugier C, Delisle MB, Rougé D, Telmon N (2014) Epifluorescence analysis of hacksaw marks on bone: highlighting unique individual characteristics. Forensic Sci. Int 241: 195-202.

24. Thompson TJ, Inglis J (2009) Differentiation of serrated and non-serrated blades from stab marks in bone. Int. J. Legal Med 123: 129-135.

25. Dooling JA, Bell WE, Jr. Whitehurst WR (1967) Penetrating skull wound from a pair of scissors. Case report. J. Neurosurg 26: 636-638.

26. Dudko S, Kusz D, Ziaja D, Kusz B (2012) Laceration of abdominal aorta by a fragment of fractured L2 vertebral body after a low-energy injury: a case report and review of literature. Spine (Phila Pa 1976) 37: 406-409.

27. Ozdemir B, Celbis O, Kaya A (2013) Cut throat injuries and honor killings: review of 15 cases in eastern Turkey. J. Forensic Leg. Med 20: 198-203.

28. Li X, Curry EJ, Blais M, Ma R, Sungarian AS (2012) Intraspinal penetrating stab injury to the middle thoracic spinal cord with no neurologic deficit. Orthopedics 35: 770-773.

29. Sharkey EJ, Cassidy M, Brady J, Gilchrist MD, NicDaeid N (2012) Investigation of the force associated with the formation of lacerations and skull fractures. Int. J. Legal Med 126: 835-844.

30. Bellamy JL, Mundinger GS, Reddy SK, Flores JM, Rodriguez ED, et al. (2013) Le Fort II fractures are associated with death: a comparison of simple and complex midface fractures. J. Oral Maxillofac. Surg 71: 1556-1562.

31. Ferllini R (2012) Macroscopic and microscopic analysis of knife stab wounds on fleshed and clothed ribs. J Forensic Sci 57: 683-690.

32. Gaudio D, Di Giancamillo M, Gibelli D, Galassi A, Cerutti E, et al. (2014) Does cone beam CT actually ameliorate stab wound analysis in bone? Int. J. Legal Med 128: 151-159. 